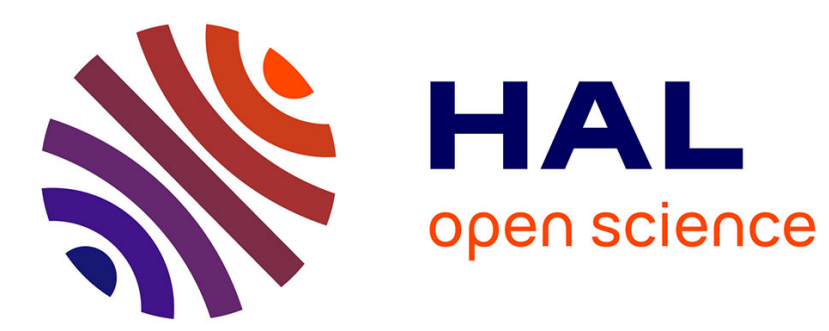

\title{
DES VERBES MODAUX PERSANS ET DE L'ÉVOLUTION DE LEUR CONSTRUCTION ENTRE LES XE ET XVIE SIĖCLES
}

Agnès Lenepveu-Hotz

\section{- To cite this version:}

Agnès Lenepveu-Hotz. DES VERBES MODAUX PERSANS ET DE L'ÉVOLUTION DE LEUR CONSTRUCTION ENTRE LES XE ET XVIE SIÈCLES. Studia Iranica, 2010, 39 (1), pp.79-107. halshs-00683036

\section{HAL Id: halshs-00683036 \\ https://shs.hal.science/halshs-00683036}

Submitted on 27 Mar 2012

HAL is a multi-disciplinary open access archive for the deposit and dissemination of scientific research documents, whether they are published or not. The documents may come from teaching and research institutions in France or abroad, or from public or private research centers.
L'archive ouverte pluridisciplinaire HAL, est destinée au dépôt et à la diffusion de documents scientifiques de niveau recherche, publiés ou non, émanant des établissements d'enseignement et de recherche français ou étrangers, des laboratoires publics ou privés. 


\title{
DES VERBES MODAUX PERSANS ET DE L'ÉVOLUTION DE LEUR CONSTRUCTION ENTRE LES $X^{\mathrm{E}}$ ET XVI ${ }^{\mathrm{E}}$ SIÈCLES
}

\begin{abstract}
RÉSUMÉ
Les verbes modaux persans bāyistan, «falloir », šāyad, «il se peut », «peut-être », tavānistan, « pouvoir », et $x^{v} \overline{a s t a n, ~ « ~ v o u l o i r ~ », ~ s e ~ c o n s t r u i s e n t, ~ s o i t ~ a v e c ~ l ' i n f i n i t i f, ~ s o i t ~ a v e c ~ u n ~}$ verbe conjugué. Comme l'étude quantitative le montre, cette double possibilité relève principalement d'une évolution. Il s'agit alors d'en chercher les causes. Excepté pour distinguer les deux emplois de $x^{v} \bar{a} s t a n$, volition et auxiliaire du futur, le sens des modalités n'est qu'une cause mineure. De même la syntaxe ne constitue pas un facteur déterminant. En réalité, ce changement de la structure régie est dû aux propriétés morphologiques du verbe conjugué, bien plus étendues que celles de l'infinitif : le verbe conjugué peut recevoir divers affixes qui sont exclus avec l'infinitif verbal.
\end{abstract}

Mots clés : verbes modaux, persan classique, diachronie, modalités, structures régies.

\section{SUMMARY}

Persian modal verbs bāyistan, "must", šāyad, "may", tavānistan, "can”, "to be able", and $x^{v} \bar{a}$ stan, "to want", get different constructions: either the infinitive or the finite verb. As the quantitative analysis shows, the choice between both constructions is principally linked to an evolution. So the causes must be looked for. Except for $x^{v} \overline{a s t a n}$ and its two uses - volition and auxiliary for the future-, the meaning of modalities takes only a little part. In the same way, syntax is not a very important feature. In fact the change of the governed construction is due to the morphological properties of the finite verb, which are much more extensive than those of the infinitive: the finite verb can get different affixes, which are excluded with the verbal infinitive.

Keywords: modal verbs, Classical Persian, diachrony, modalities, governed constructions.

\section{Introduction}

La structure syntaxique des verbes modaux bāyistan, «falloir », šāyad, « il se peut », « peut-être », tavānistan, «pouvoir », et $x^{v} \bar{a} \operatorname{stan}$, «vouloir », connaît une évolution sensible 
entre les débuts du persan, au $\mathrm{X}^{\mathrm{e}}$ siècle, et le persan contemporain d'Iran ${ }^{1}$. Le plus souvent ${ }^{2}$ ces verbes se construisent avec un infinitif, plein ou apocopé, à époque ancienne, alors qu'en persan contemporain, le verbe régi est généralement conjugué au mode subjonctif. Pour deux d'entre eux, bāyistan et tavānistan, lorsque le verbe régi est à l'infinitif en persan d'aujourd'hui, c'est que la tournure est impersonnelle: «falloir ${ }^{3}$ et «pouvoir» ne concernent pas un individu particulier mais renvoient à un indéfini. Comme l'infinitif alterne avec un verbe conjugué aussi bien dans les textes anciens que dans l'impersonnel actuel, il s'agit d'un infinitif verbal et non pas d'un infinitif nominal ${ }^{4}$.

Cette évolution, souvent évoquée dans les grammaires n'a, à ma connaissance, jamais fait l'objet d'une étude qui tenterait d'en établir les causes. C'est ce que je me propose de chercher. Pour cela, je commencerai par l'étude des proportions de l'une et l'autre structures aux différentes époques, $\mathrm{X}^{\mathrm{e}}-\mathrm{XI}^{\mathrm{e}}$ siècles et $\mathrm{XV}^{\mathrm{e}}-\mathrm{XVI}^{\mathrm{e}}$ siècles, dans un corpus de six textes. Je comparerai des occurrences avec les deux constructions issues d'un même texte, quand cela est possible. De là apparaîtront les facteurs qui orientent l'énonciateur à opter pour une structure plutôt que l'autre : le sens des diverses modalités exprimées par ces verbes, la syntaxe de la structure qu'ils régissent et l'emploi de certains morphèmes.

\subsection{Auxiliarisation}

Il nous faut d'abord réfléchir sur la nature de ces verbes : sont-ils des auxiliaires ou non? Pour y répondre, je me réfèrerai aux critères de Denis Creissels (1995: 180) sur l'auxiliarisation.

Selon le premier critère, l'auxiliant «présente à lui seul les caractéristiques morphologiques d'une forme verbale». Si tavānistan, «pouvoir», et $x^{v} \bar{a} s t a n$, « vouloir», peuvent se fléchir en personne, ce n'est pas le cas des impersonnels bāyistan, «falloir», et $\check{s} \bar{a} y a d$, , il est possible », qui, eux, sont des verbes défectifs. Cette caractéristique n'est donc pas valide pour ces deux derniers verbes.

Leur verbe régi doit également être un « lexème fonctionnant par ailleurs comme base de formes verbales simples ». Nos quatre verbes se plient à ce critère.

Enfin pour Creissels, il est nécessaire que «l'ensemble "auxiliant + auxilié" [...] s'analys[e] globalement comme un terme prédicatif unique totalement équivalent, en ce qui

\footnotetext{
${ }^{1}$ Certaines des anciennes structures se trouvaient encore dans le persan parlé en Afghanistan au début du XX ${ }^{\mathrm{e}}$ siècle. Cf. Phillott 1919, p. 252 pour tavānistan par exemple.

${ }^{2}$ Cette appréciation sur la fréquence, partagée comme allant de soi par la majorité des grammaires, sera bien évidemment à nuancer.

${ }^{3}$ Cf. Bāteni 1354/1975, p. 197.

${ }^{4}$ Voir sur cette distinction Mir-Samii et Samvelian 2007, p. 126.
} 
concerne sa valence, à une forme verbale simple issue du lexème identifiable dans l'auxilié ». De nouveau seuls tavānistan, «pouvoir », et $x^{v} \bar{a} s t a n$, «vouloir», répondent à cette définition, et dans le cas de $x^{v} \bar{a}$ stan que ce soit dans son sens premier de volition, alors auxiliaire modal, ou comme auxiliaire temporel du futur. Cette condition ne s'applique pas à chacun de nos verbes.

Ces verbes ne pouvant tous être considérés comme des auxiliaires, il me paraît plus prudent d'en parler comme verbes modaux et non comme auxiliaires modaux. De plus, bien que ces verbes n'aient pas les mêmes propriétés syntaxiques et morphologiques, je les analyserai ensemble : c'est le même facteur qui conditionne l'évolution de leur structure.

\subsection{Le corpus}

Je me suis appuyée sur de larges extraits ${ }^{5}$ de six textes : trois des $\mathrm{X}^{\mathrm{e}}-\mathrm{XI}^{\mathrm{e}}$ siècles et trois des $\mathrm{XV}^{\mathrm{e}}-\mathrm{XVI}^{\mathrm{e}}$ siècles. Il s'agira de comparer les deux états de langue.

- HM : Abū Bakr Rabī‘ b. Ahmad al-Axavainī al-Buxārī, Kitāb hidāyat al-muta 'allimīn fì altibb (vers 370/980), éd. J. Matini, Mašhad, 1344/1965, p. 13-200.

- TE : Tafsīr d'Ezéchiel (déb. $11^{\mathrm{e}}$ s.), éd. T. E. Gindin, Wien, 2007. Ce texte présente deux dialectes distincts, nord-est et sud-ouest (Khuzistān ?). Ont été étudiées les pages du manuscrit 1-20 et 130-150 pour le premier dialecte, et les pages 170-190 et 195-215 pour le second.

- TS : Tārīx-i Sīstān (1053-63), éd. M. T. Bahār, Tehrān, 1314/1935, p. 1-200.

- PR : Hāfiz-i Abrū, Panj risāla-i tārīxī (1414-36), éd. F. Tauer, Prague, 1959, 68 p.

- RA : Amīr Jamāl al-Dīn 'Atā’ Allāh b. Fazl Allāh al-Husainī al-Daštakī al-Šīrāzị, Rauzat alAhbāb fī siyar al-Nabī wa-'l-Āl wa-'l-Ashāb (888/1484), manuscrit présenté comme autographe, conservé à la bibliothèque Astān-e Qods de Mašhad, p. 1-50 et 597-646.

- SX : Nūr al-Dīn Muhammad b. Husain b. Abd Allāh b. P̄̄r Husain b. Šams al-Dīn al-Qazvīnī, Silsila-nāma-i x $x^{v} \bar{j} j a g \bar{a} n-i$ naqšband (978/1570-1), manuscrit de 983/1585, conservé à la Bibliothèque nationale de France, 44 p.

\section{3. Répartition de ces verbes construits avec infinitif ou verbe conjugué}

Pour ne pas fausser les pourcentages, je n'ai comptabilisé les occurrences que dans des passages de longueur quasi-équivalente, à savoir 70-100 pages (en fonction du nombre de lignes par page). Ainsi, j'ai ramené HM aux pages 100 à 200 et TS aux pages 1 à 100 . Et bien

\footnotetext{
${ }^{5}$ Les passages varient d'environ 70 à 200 pages selon le texte, sa nature, son nombre de lignes par page, excepté pour le dernier qui ne compte qu'une quarantaine de pages et qui bien sûr a été analysé dans son intégralité.
} 
sûr les occurrences où ces verbes sont employés de manière isolée ne sont pas prises en compte.

bāyistan, « falloir »

\begin{tabular}{|l|c|c|c|}
\hline & Infinitif & Verbe conjugué & \% Infinitif / Total \\
\hline HM (vers 980) & 37 & 5 & $88 \%$ \\
\hline TE (déb. $11^{\mathrm{e}}$ s.) & & $2 / 6$ & $20 \%(50 \% / 0 \%)$ \\
\hline TS (1053-63) & $2 / 0$ & 4 & $84 \%$ \\
\hline PR (1414-36) & 21 & 6 & $60 \%$ \\
\hline RA (1484) & 9 & 18 & $31 \%$ \\
\hline SX (1570-1) & 1 & 0 & $(100 \%)^{7}$ \\
\hline
\end{tabular}

Le texte judéo-persan TE mis à part, nous pouvons constater que la proportion de constructions infinitives avec le verbe bāyad, «falloir », tend à décroître du $\mathrm{X}^{\mathrm{e}}$ au XVI $\mathrm{X}^{\mathrm{e}}$ siècles, avec une forte diminution (environ de moitié) entre le début et la fin du $\mathrm{XV}^{\mathrm{e}}$ siècle.

$\check{s} \bar{a} y a d$, « il se peut », «peut-être »

\begin{tabular}{|l|c|c|}
\hline & Infinitif & Verbe conjugué \\
\hline HM (vers 980) & 1 & 0 \\
\hline TE (déb. $11^{\mathrm{e}}$ s.) & $7 / 5$ & $7 / 0$ \\
\hline TS $(1053-63)$ & 1 & 0 \\
\hline PR (1414-36) & 0 & 0 \\
\hline RA (1484) & 0 & 2 \\
\hline SX (1570-1) & 1 & 0 \\
\hline
\end{tabular}

Le peu d'occurrences de šāyad ne permet aucune analyse. Seuls bāyistan, «falloir », tavānistan, « pouvoir », et $x^{v} \bar{a} s t a n$, « vouloir », seront alors étudiés.

tavānistan, « pouvoir»

\begin{tabular}{|l|c|c|c|}
\hline & Infinitif & Verbe conjugué & \% Infinitif / Total \\
\hline HM (vers 980) & 38 & 0 & $100 \%$ \\
\hline TE (déb. $11^{\mathrm{e}}$ s.) & $8 / 2$ & $1 / 2$ & $76,9 \%\left(88,9 \% / 50 \%{ }^{8}\right)$ \\
\hline
\end{tabular}

\footnotetext{
${ }^{6}$ Étant donné les deux dialectes de TE, les nombres d'occurrences de chacun seront distingués par/.

${ }^{7}$ Les pourcentages dans ce texte moins long que les autres seront indiqués entre parenthèses.
} 


\begin{tabular}{|l|c|c|c|}
\hline TS (1053-63) & 7 & 0 & $100 \%$ \\
\hline PR (1414-36) & 10 & 0 & $100 \%$ \\
\hline RA (1484) & 26 & 4 & $86,7 \%$ \\
\hline SX (1570-1) & 8 & 1 & $(88,9 \%)$ \\
\hline
\end{tabular}

Il faut laisser à nouveau de côté TE pour percevoir une évolution : tavānistan, «pouvoir », se construit exclusivement avec l'infinitif aux $\mathrm{X}^{\mathrm{e}}-\mathrm{XI}^{\mathrm{e}}$ siècles comme encore au début $\mathrm{du} \mathrm{XV}^{\mathrm{e}}$ siècle ${ }^{9}$ puis il régit aussi un verbe conjugué dans les deux derniers textes du corpus. C'est dire que l'évolution se noue au cours du XV siècle. En outre, si l'on excepte les cas où tavānistan avec infinitif est à la forme impersonnelle tavān, la proportion pour RA n'est plus que de $82,6 \%$ et pour SX de $66,7 \%$. La construction avec verbe conjugué ne se réduit donc pas à l'état d'exception dans ces textes.

$x^{v} \bar{a}$ stan, « vouloir » ${ }^{10}$

\begin{tabular}{|l|c|c|c|}
\hline & Infinitif & Verbe conjugué & \% Infinitif / Total \\
\hline HM (vers 980) & $1+2$ & 5 & $37,5 \%$ \\
\hline TE (déb. $11^{\mathrm{e}}$ s.) & $1+1 / 3+1$ & $14+2 / 13$ & $17,1 \%(11,1 \% / 23,5 \%)$ \\
\hline TS (1053-63) & 3 & 20 & $13 \%$ \\
\hline PR (1414-36) & $4+5$ & 12 & $42,9 \%$ \\
\hline RA (1484) & 1 & 28 & $3,4 \%$ \\
\hline SX (1570-1) & 0 & 1 & $(0 \%)$ \\
\hline
\end{tabular}

A première vue, l'évolution de la structure paraît ici moins nette que pour les autres verbes. Pour PR, si l'on excepte les 5 hésitations signalées dans la première colonne, le pourcentage serait alors de $25 \%$. On aurait ainsi une proportion d'infinitif qui varierait dans un large intervalle (de $11,1 \%$ à 37,5 \%) pour les textes jusqu'à $P R$ puis qui chuterait à partir de RA (le faible pourcentage correspond en réalité à une seule occurrence, de surcroît douteuse). Si cette évolution n'est pas linéaire, c'est qu'il s'agit peut-être de nuances dialectales entre les trois textes plus récents mais elles sont moins sensibles que dans les premiers textes.

\footnotetext{
${ }^{8}$ Une occurrence est douteuse : il se peut qu'il y manque un verbe à l'infinitif avant le verbe conjugué. La proportion dans le deuxième dialecte passerait de $50 \%$ à $75 \%$, saut quantitatif très important eu égard au faible nombre d'occurrences. Elle n'est donc pas exploitable.

${ }^{9}$ Nous verrons que c'est déjà la situation en moyen perse.

${ }^{10}$ Dans les deux tableaux suivants, j'ai séparé des autres occurrences par le signe + les cas où il est impossible de trancher entre volition et futur, et cela, même en fonction du contexte.
} 
$x^{v} \bar{a} \operatorname{stan}$, auxiliaire du futur

\begin{tabular}{|l|c|c|c|}
\hline & Infinitif & Verbe conjugué & \% Infinitif / Total \\
\hline HM (vers 980) & $8+2$ & 0 & $100 \%$ \\
\hline TE (déb. 11 ${ }^{\mathrm{e}}$ s.) & $18+1 / 12+1$ & $9+2 / 0$ & $74,4 \%(63,3 \% / 100 \%)$ \\
\hline TS (1053-63) & 8 & 0 & $100 \%$ \\
\hline PR (1414-36) & $25+5$ & 0 & $100 \%$ \\
\hline RA (1484) & 41 & 0 & $100 \%$ \\
\hline SX (1570-1) & 1 & 0 & $(100 \%)$ \\
\hline
\end{tabular}

Hormis dans le premier dialecte de TE, le verbe $x^{v} \bar{a}$ stan se construit exclusivement avec l'infinitif lorsqu'il sert à former le futur.

\begin{tabular}{|l|c|c|}
\hline$\%$ Volition / Total & Infinitif & Verbe conjugué \\
\hline HM (vers 980$)$ & $27,3 \%$ & $100 \%$ \\
\hline TE (déb. $11^{\mathrm{e}}$ s.) & $16,7 \%(10 \% / 25 \%)$ & $76,3 \%(64 \% / 100 \%)$ \\
\hline TS $(1053-63)$ & $27,3 \%$ & $100 \%$ \\
\hline PR (1414-36) & $26,5 \%$ & $100 \%$ \\
\hline RA (1484) & $2,4 \%$ & $100 \%$ \\
\hline SX (1570-1) & $(0 \%)$ & $(100 \%)$ \\
\hline
\end{tabular}

Lorsque $x^{v} \bar{a}$ stan se construit avec un verbe conjugué, il indique exclusivement la volition, à l'exception encore du premier dialecte de TE. Avec l'infinitif, il représente environ un quart des occurrences jusqu'au début de $\mathrm{XV}^{\mathrm{e}}$ siècle (sauf dans le premier dialecte de TE) puis dix fois moins à la fin de ce même siècle.

Au vu de ces données chiffrées, il apparaît clairement que les proportions sont plus ou moins stables entre le $\mathrm{X}^{\mathrm{e}}$ siècle et le début du $\mathrm{XV}^{\mathrm{e}}$ siècle. À partir de RA, les structures commencent à changer, toujours au profit de la construction avec verbe conjugué. Il faut cependant nuancer ce constat en soulignant qu'il ne se fonde que sur un seul texte, même si SX, pas assez long pour être entièrement significatif, paraît confirmer cette tendance.

TE est souvent plus proche de l'état de langue du XVI $\mathrm{XI}^{\mathrm{e}}$ siècle qu'il ne l'est des textes de son époque, surtout pour le premier dialecte. Cela est d'autant plus étonnant que ce premier dialecte émane du nord-est, c'est-à-dire de la même région que les textes en écriture arabe des

\footnotetext{
${ }^{11}$ Les hésitations entre expression de la volonté et futur ont été comptabilisées dans la volition, en privilégiant le sens premier de $x^{v} \bar{a} s t a n, \ll$ vouloir ».
} 
premiers siècles. Peut-être faudrait-il alors l'apparenter aux dialectes du nord-ouest, comme le propose Gilbert Lazard ${ }^{12}$ ? L'étude d'autres textes judéo-persans s'avèrerait nécessaire pour clarifier ce point.

\section{Sens de la modalité}

Si l'on se réfère à la typologie de Frank R. Palmer ${ }^{13}$, les deux premiers verbes modaux étudiés sont susceptibles d'exprimer diverses modalités. Ainsi selon les occurrences, bāyad, « il faut », exprime la modalité déontique obligative (l'énonciateur conçoit l'événement comme obligatoire selon des facteurs externes) ou la modalité épistémique déductive (l'énonciateur conçoit l'événement comme nécessaire en fonction d'un raisonnement, comme sa seule conclusion possible); et tavānistan, «pouvoir», exprime, soit la modalité dynamique capacitive (l'énonciateur conçoit l'événement comme possible selon des facteurs internes de capacité), soit la modalité déontique permissive (l'énonciateur conçoit l'événement comme permis selon des facteurs externes), soit la modalité épistémique spéculative (l'énonciateur conçoit l'événement comme possible en fonction d'un raisonnement, comme une conclusion possible). Il est tout à fait légitime de croire que ces distinctions de valeur peuvent avoir une influence sur la structure régie par le verbe modal.

De la même façon que la sémantique des verbes modaux conditionne peut-être leur construction, j'étudierai aussi les différences de structures pour $x^{v} a \overline{s t a n}$ selon qu'il est l'expression de la volition ou l'auxiliaire du futur.

\section{1. bāyad, modalité déontique obligative et modalité épistémique déductive}

Pour la modalité déontique obligative, les deux structures se rencontrent indifféremment, quels que soient les textes et les époques. Dans un texte ancien comme TE, le verbe se construit avec l'infinitif en (1a) et avec un verbe conjugué en (1b), de même au $\mathrm{XV}^{\mathrm{e}}$ siècle, dans PR par exemple, en (2).

(1) a. $\check{c} i$

hamē bāyast

abāz guftan ${ }^{14}$

pourquoi $m \bar{e}$ falloir.PAS.3SG PREV dire-INF ${ }^{15}$

\footnotetext{
${ }^{12}$ Cf. Édition Gindin 2007, I, p. 26 note 107.

${ }^{13}$ Palmer 2001, p. 22, et pour le persan contemporain Rahimian 1999 et Taleghani 2008a et 2008b. J'ai parfois dû franciser les termes de la terminologie anglo-saxonne.

${ }^{14} \mathrm{~J}$ 'ai opté pour une transcription des caractères arabes, et non une translittération (ex : $\bullet$ et $b$ transcrits t). Les voyelles seront transcrites sous leur forme classique, même si l'articulation de certaines a évolué au XVI ${ }^{\mathrm{e}}$ siècle. Le judéo-persan sera transcrit comme les textes en écriture arabe.
} 
pourquoi était-ce nécessaire de répéter ? (TE 1,14$)^{16}$

\section{b. bāalastēe ki šumā saxtīhā abar $x^{v} \bar{e} \bar{s} t a n$ avardēdēe}

falloir.PAS.3SG-ē que vous malheur-PL sur REFL apporter.PAS-2PL-ē

il fallait que vous apportiez les malheurs sur vous-mêmes (TE 12, 40)

(2) a. bar har sar-i kūyē čand kas-rā mēbāyad kušt

sur chaque tête-EZ rue-IND quelque personne-rā $m \bar{e}$ falloir.PST-3SG tuer.INF

il faut en tuer plusieurs à l'entrée de chaque rue (PR 64, 25 - 65, 1)

b. mē bāalad ki $\bar{u}-r \bar{a}$ girifta biyāvarē

$m \bar{e}$ falloir.PST-3SG que lui-rā prisonnier $b i$-apporter.PST-2SG

il faut que tu (me) l'amènes prisonnier (PR 14, 9)

La modalité épistémique déductive, elle, ne semble s'exprimer qu'avec bāyad suivi d'un verbe régi conjugué, et ce aux deux époques. PR et SX ne présentent aucune occurrence de cette modalité. Remarquons d'ailleurs qu'on ne trouve dans tous ces textes qu'un nombre extrêmement faible d'occurrences (4, à savoir une par texte). Il est donc impossible de tirer des conclusions. On ne peut parler ici que d'une tendance de la langue.

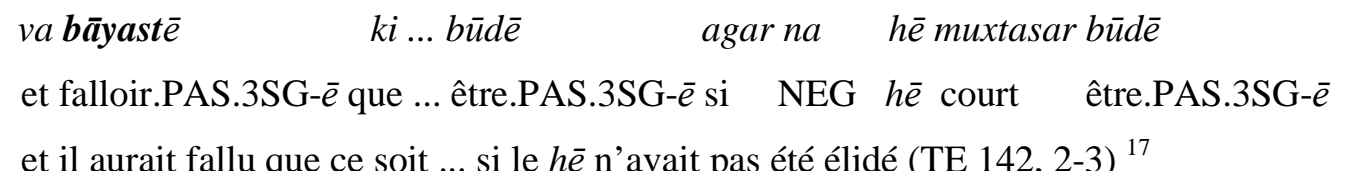

et il aurait fallu que ce soit ... si le hē n'avait pas été élidé (TE 142, 2-3) ${ }^{17}$
dartab'-i
havā-i $\bar{o}$
maujūd ast
ki $\quad \bar{a} n j \bar{a} n \bar{a} \bar{c} a \bar{r}$
'ulamā- $i$

dans nature-EZ climat-EZ lui existant être.PST.3SG que là nécessairement savant.PL-EZ

bisyār bāyad ki bāšad va 'āmma-i Sistān 'ilm dōst

beaucoup falloir.PST-3SG que être.PST-3SG et peuple-EZ Sistān science ami

$$
\text { bāayad } \quad k i \quad b \bar{a} \check{s} a d
$$

falloir.PST-3SG que être.PST-3SG

la nature de son climat est telle qu'il doit nécessairement y avoir beaucoup de savants et que le peuple du Sistān doit aimer la science (TS 13, 5-6)

\footnotetext{
${ }^{15}$ Liste des abréviations en fin d'article.

${ }^{16}$ Entre parenthèses : la page et la ligne de l'exemple choisi, parmi d'autres occurrences du même type.

${ }^{17}$ En judéo-persan : ... correspond à un ou plusieurs mots hébreux.
} 
La modalité épistémique déductive est renforcée par l'expression de la cause de la déduction dans 3 de ces occurrences et pour 2 d'entre elles, elle est présentée par une conditionnelle introduite par agar, «si », par exemple en (3).

2.2. tavānistan, modalité dynamique capacitive, modalité déontique permissive ${ }^{18}$ et modalité épistémique spéculative

Je me suis fondée sur les textes où les deux structures sont attestées conjointement, à savoir TE, RA et SX.

La modalité dynamique capacitive est la modalité la plus fréquente dans notre corpus. Elle s'exprime dans ces trois textes aussi bien par un infinitif (5) que par un verbe conjugué (6).

(5) a. ki natavāne e abar suxanān-i ēěs̄an vaqf būdan que NEG-pouvoir.PST-2SG sur parole-PL-EZ eux comprendre-INF de qui tu ne peux pas comprendre les paroles (TE 6, 33-34)

b. pindāštt ki ba īn tadbīr taqyīr-i taqdīr-i ilāhī tavān penser.PAS.3SG que à ce mesure changement-EZ destin-EZ divin pouvoir.PST.IMP faire.INF il pensait qu'avec cette mesure on peut changer le destin divin (RA 31, 1-2)

c. xasāyis-i Šām vafazāyil-i ān maqām čigūna šarh caractéristique.PL-EZ Damas et qualités.PL-EZ ce lieu saint comment description tavān $d \bar{a} d$ pouvoir.PST.IMP donner.INF les caractéristiques de Damas et les qualités de ce lieu saint, comment peut-on (les) décrire (SX 40) ${ }^{19}$

(6) a. natavāne

ki bišinavēe suxanān-i ēšān

NEG-pouvoir.PST-2SG que $b i$-entendre.PST-2SG parole-PL-EZ eux

tu ne peux pas entendre leurs paroles (TE 6,37)

b. agar mē tavānē

ki bihtar az īn biyāfarīnē

biyāaraīn

si $\quad m \bar{e}$ pouvoir.PST-2SG que mieux que ceci $b i$-créer.PST-2SG $b i$-créer.IMPER.2SG

\footnotetext{
${ }^{18}$ Aucun exemple ne pouvant s'analyser de façon sûre comme expression de la modalité déontique permissive, cette modalité sera laissée de côté.

${ }^{19}$ La présentation particulière de ce texte - non linéaire mais "en étoile" - ne permet pas de numéroter les lignes.
} 
si tu es capable de créer mieux que ceci, crée(-le) (RA 24, 20)

c. mētavānad ki kunad rāh namāȳ

$m \bar{e}$ pouvoir.PST-3SG que faire.PST-3SG chemin action de montrer

il peut montrer le chemin (SX 36)

J'ai choisi les exemples très proches (5a) et (6a) pour montrer qu'ici ce n'est pas la modalité qui induit le choix de la structure. Les termes se retrouvent presque mot pour mot dans ces deux occurrences. J'y reviendrai.

Pour exprimer la modalité épistémique spéculative, le verbe modal tavānistan se construit avec un verbe à l'infinitif. On en trouve beaucoup d'exemples dont (7). Seul (8) présente un verbe conjugué. Encore cette occurrence est-elle contestable puisqu'il est possible que le verbe guftan ait été omis, volontairement ou non, après tavānē. Elle se comprendrait alors comme $\ll$ tu ne peux pas dire que ce soit... ${ }^{20}$.

pastavānad būd ki Idrīs 'arabì bovad

donc pouvoir.PST-3SG être.INF que Idrīs arabe être.PST-3SG

donc il se peut que Hénoch soit arabe (RA 22,12)

(8)

in natavāne $\overline{\boldsymbol{e}} \quad k i \ldots \quad b \bar{u} d \bar{e}$

ceci NEG-pouvoir.PST-ē que ... être.PAS.3SG-ē

ceci ne peut pas être... (TE 176, 33-34)

\section{3. $\mathrm{x}^{\mathrm{V}}$ āstan, expression de la volition et auxiliaire du futur}

Comme les proportions l'ont déjà mis en évidence, les structures avec $x^{v} a \overline{s t a n}$, « vouloir », dépendent du sens de ce verbe pour une grande partie des occurrences. Il peut en effet exprimer la volition ou bien servir à la tournure périphrastique du futur. Gilbert Lazard (2006a : 63) rappelle d'ailleurs que futur comme volition relèvent d'une même catégorie : « the common feature of all kinds of irrealis (possible, intentional, (un)desirable, (un)necessary, future, etc.), in contradistinction to the realis, is that the actualisation of the event is uncertain ». Ainsi dans une perspective diachronique, l'utilisation de la volition pour former le futur se retrouve dans beaucoup de langues : par une périphrase comme dans l'anglais I will come ou dans la morphologie même, par exemple en grec ancien sur un thème de désidératif en

\footnotetext{
${ }^{20}$ Cf. Édition Gindin 2007, II, p. 365 note 693.
} 
*-se/o- ${ }^{21}$. Pour ce qui est du passage de l'un à l'autre en persan, on peut aisément penser avec Carina Jahani (2008 : 155 et 165) que, de la volition, on serait passé au futur d'intention, puis au futur de prédiction.

Dès nos premiers textes, nous constatons une tendance à utiliser la structure avec verbe conjugué pour marquer la volition, ce qui la distingue de l'expression du futur, avec verbe à l'infinitif ${ }^{22}$. Dans les exemples (9a) et (9b), seul diffère le sens de $x^{v} \bar{a} s t a n$, les compléments, eux, sont de même type.

(9) a. $x^{v} \bar{a} h a d$

ki adab $\quad \bar{a} m \bar{z} z a d$

ba āsān̄

vouloir.PST-3SG que instruction apprendre.PST-3SG à facilité

il veut acquérir de l'instruction sans effort (TS 106, 8)

b. man šahrēe banā $\quad \boldsymbol{x}^{v} \bar{a} h a m \quad$ kard badīn rōzgārki...

je ville-IND construction vouloir.PST-1SG faire.INF à-ce époque que...

je construirai une ville à cette époque où... (TS 3,9)

Rappelons qu'il ne s'agit que d'une tendance de la langue et tendance n'est pas règle. On comprendra alors pourquoi Douglas C. Phillott n'a vu aucune différence entre les deux, affirmant que $x^{v} \bar{a}$ stan est suivi de l'infinitif apocopé pour le futur, comme pour la volition ${ }^{23}$. Encore nous faut-il utiliser son propos avec précaution puisque l'auteur ne s'appuie que sur des exemples en vers tirés du Gulīstān de Sa'dī. Et l'on sait combien, exploitant le génie de la langue jusqu'à ses dernières ressources, le poète s'autorise des libertés grammaticales pour respecter la métrique. Mais il est vrai que même dans la prose, on peut aussi rencontrer un infinitif avec l'idée de volonté, ainsi en est-il en (10). En revanche la structure avec verbe conjugué marque exclusivement la volition, excepté dans quelques occurrences du premier dialecte de TE.

$\check{c ̌ u n}^{24}$ dast-i rāst $\boldsymbol{x}^{v} \bar{a}$ had gaštan

quand main-EZ droite vouloir.PST-3SG tourner-INF

quand il veut tourner la main droite (litt. la main droite veut tourner) (HM 59, 11-12)

\footnotetext{
${ }^{21}$ Sur ce lien entre volition et futur, voir Comrie 1985, p. $45-46$ et pour le grec, Chantraine 1991, p. 246-247.

${ }^{22}$ Ce point a déjà été relevé par Ludwig Paul (2002: 26).

${ }^{23}$ Phillott 1919, p. 258-259.

${ }^{24}$ Le mot est écrit sans $v \bar{a} v$ ( و g), c'est-à-dire que le /u/ est bref.
} 
Comme le montre le premier tableau des relevés de $x^{v} \bar{a}$ stan, cette tendance à distinguer les deux emplois par le verbe régi ira en s'accentuant (de 37,5\% dans HM à 3,7 \% dans RA pour la volition exprimée avec une structure infinitive, sur le total de la volition dans ces textes). Ainsi dans un même texte (PR), constate-t-on un remaniement des formes entre d'une part les manuscrits $\mathrm{M}_{1}$ et $\mathrm{M}_{2}$ (le premier antérieur à 850/1447, le second daté de 885/1480) et d'autre part trois manuscrits $\mathrm{G}$ dont le plus ancien date de $1600{ }^{25}$. Dans (11a), $x^{v} \bar{a} s t a n$ exprimant la volition régit des infinitifs alors que dans (11b), il régit des verbes conjugués. Or si l'on regarde plus attentivement les dates de ces manuscrits, on retrouve le laps de temps qui sépare PR de RA, laps de temps au cours duquel précisément nous avions vu que la tendance à utiliser un verbe régi conjugué s'accentuait.

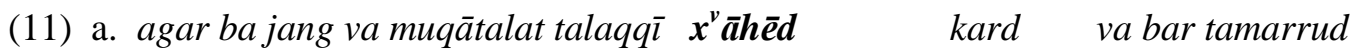

si à guerre et combat accueil vouloir.PST-2PL faire.INF et sur rébellion

isrār namūd va bahisānat-i qal'a va dīvārpanāhīd

obstination montrer.INF et à solidité-EZ forteresse et mur réfugier.INF

si vous voulez traiter (cela) par la guerre et le combat acharné, persister dans la rébellion et vous réfugier à l'abri de la forteresse et de (ses) murs (PR 65, 5-6) manuscrits $\mathrm{M}_{1}$ et $\mathrm{M}_{2}$

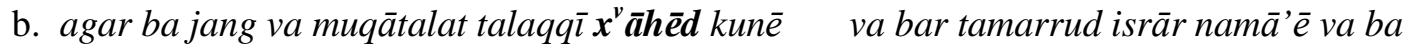
faire.PST-2SG montrer.PST-2SG

hisānat-i qal 'a va dīvār maqrūr šave ${ }^{26}$

fier devenir.PST-2SG

si vous voulez traiter (cela) par la guerre et le combat acharné, persister dans la rébellion et tirer fierté de la solidité de la forteresse et de (ses) murs (PR 65, 5-6) manuscrits G

Si la répartition s'est faite ainsi entre les deux structures, on peut penser que c'est aussi pour une raison sémantique. Faut-il y voir, comme l'envisage Palmer pour d'autres langues (2001 : 192-196), que l'emploi d'un verbe conjugué (au subjonctif ? ${ }^{27}$ ) marque un plus haut degré d'irréalité que l'infinitif ? Très certainement. La distinction entre futur et volonté ne réside-t-elle pas en effet entre un événement senti comme réalisable dans l'avenir et un

\footnotetext{
${ }^{25}$ Voir l'introduction de l'édition de Tauer, p. X-XV.

${ }^{26}$ Il semble y avoir une erreur de terminaison : $x^{v} \bar{a} h \bar{e} d$ est à la deuxième personne du pluriel tandis que kune, $n a m \bar{a} ' \bar{e}$ et $\breve{s} a v \bar{e}$ sont à la deuxième personne du singulier. Même si l'on envisage un cas de non-coréférence, la phrase n'aurait pas de sens. Ou faut-il voir ici la variante $-\bar{e}$, marque de deuxième personne du pluriel attestée dans les textes anciens et dans certains parlers tadjiks actuels ? Cf. Lazard 1963, p. 267-269.

${ }^{27}$ En persan classique, il n'est pas possible de distinguer morphologiquement le subjonctif de l'indicatif. Mais le mode utilisé en persan contemporain avec $x^{v} \bar{a} s t a n$, « vouloir », est bien le subjonctif.
} 
événement que l'on aimerait voir se réaliser mais dont on ne sait s'il se produira réellement dans l'avenir?

\section{Considérations syntaxiques}

Avec un verbe conjugué, on s'attend à ce que la phrase ait davantage la possibilité de se développer qu'avec un verbe à l'infinitif où la structure régie et celle du verbe modal ne sont pas toujours distinctes. Il conviendra alors d'analyser la place et la voix des verbes régis ainsi que les compléments qui en dépendent.

\subsection{Place de l'infinitif}

Pour la structure régie par le verbe modal, l'énonciateur est face à une double option : infinitif et verbe conjugué d'une part, infinitifs antéposé et postposé au verbe modal d'autre part. Dans notre corpus, l'infinitif qui dépend du verbe modal est antéposé à ce dernier pour 6 occurrences. On trouve cette structure avec tous les verbes modaux : bāald dans HM 17, 11 ; tavānistan dans HM 136, 15 ; TE 7, 5 ; TS 114, 9; et $x^{v} \bar{a}$ stan dans HM 142, note 11 (dans le manuscrit F datant de 510/1116); RA 36, $17^{28}$.

Or nous savons que cette construction n'est pas rare en moyen perse. Christopher Brunner en fait même la structure habituelle pour les verbes impersonnels en proposant le schéma suivant :

«['agent'] [object of infinitive] complementary infinitive impersonal verb, inflected ${ }^{29}$

Si l'on inventorie la construction des verbes modaux dans le texte moyen-perse Kārnāmag $\bar{l}$ Ardax̌̌ēe $\bar{l}$ Pābagān, on constate que les infinitifs leur sont autant antéposés que postposés $^{30}$ :

\begin{tabular}{|c|c|c|c|}
\hline & Infinitif antéposé & Infinitif postposé & $\begin{array}{c}\text { Verbe conjugué } \\
\text { introduit par } k \bar{u}\end{array}$ \\
\hline abāyistan & 2, soit $15,3 \%$ & 10, soit $77 \%$ & 1, soit $7,7 \%$ \\
\hline šäystan & 10, soit $52,6 \%$ & 8, soit $42,1 \%$ & 1, soit $5,3 \%$ \\
\hline tuwān & 4, soit $50 \%$ & 4, soit $50 \%$ & 0 \\
\hline
\end{tabular}

\footnotetext{
${ }^{28}$ Pour des exemples tirés d'autres textes, voir Lazard 1963, p. 351.

${ }^{29}$ Brunner 1977, p. 189.

${ }^{30}$ On remarquera aussi que la construction avec verbe conjugué n'est presque pas employée dans ce texte, et ne l'est jamais avec tavānistan, ce qui confirme l'ancienneté de la structure infinitive sur le verbe régi conjugué.
} 
D'après les occurrences recensées par Desmond Durkin-Meisterernst, nous retrouvons sensiblement cette même répartition dans les textes moyens-perses manichéens :

\begin{tabular}{|c|c|c|c|}
\hline & Infinitif antéposé & Infinitif postposé & $\begin{array}{c}\text { Verbe conjugué } \\
\text { introduit par } k \bar{u}\end{array}$ \\
\hline abāyistan & 0 & 1 & 0 \\
\hline šàistan & 3 & 1 & 0 \\
\hline tuwān & 0 & 4 & 1 \\
\hline
\end{tabular}

La présence de cette structure en persan serait donc une survivance de l'ancienne construction, déjà en concurrence avec l'infinitif postposé en moyen perse. Sur les 6 occurrences, 3 apparaissent dans le plus ancien de nos textes, HM, ce qui tendrait à confirmer mon hypothèse. Toutefois, la structure est encore attestée dans le persan parlé en Afghanistan et en Inde au début du $\mathrm{XX}^{\mathrm{e}}$ siècle ${ }^{31}$, persan qui, nous le savons, conserve certains archaïsmes.

Mais y a-t-il entre ces occurrences et celles où l'infinitif est postposé une distinction de sens ou de structure ? Pas réellement. Ainsi avec bāyad, la structure ne diffère pas entre (12a) et (12b) : les verbes y régissent les mêmes compléments (objet direct défini et circonstant).

(12) a. har yakēerā ba kārburdan ba jāygā̄h-i dīgar bāyad

chaque un-IND-rā utiliser-INF à place-EZ autre falloir.PST-3SG

il faut utiliser chacun à une autre place (HM 17, 11)

b. ìn suxanān ìnjā yād bäayad kan

ce parole-PL ici évocation falloir.PST-3SG faire-INF

il faut évoquer ces paroles ici (HM 93, 10)

Pour tavānistan, cette différence n'existe pas non plus entre (13a) et (13b) où le même verbe à l'infinitif est employé. Cependant la présence de yārī natavānad kardan en (13a), c'està-dire d'un premier verbe tavānistan, a pu modifier la structure. On retrouve deux verbes tavānistan avec infinitifs antéposé puis postposé dans (13c): en plus d'apporter un effet stylistique de chiasme, l'énonciateur y évite la confusion entre les deux propositions.

(13) a. garmī-i jigar yārī natavānad kardan va muqāvamat kardan chaud-ī-EZ foie aide NEG-pouvoir.PST-3SG faire-INF et solidité faire-INF

\footnotetext{
${ }^{31}$ Phillott 1919, respectivement pour bāyad, tavānistan et $x^{v} \bar{a}$ stan p. 268, 250 et 259.
} 
natavānad mar sardī-i dil-rā

NEG-pouvoir.PST-3SG mar froid-ī-EZ coeur-rāa

le chaud du foie ne peut pas aider et ne peut pas stabiliser le froid du coeur (HM 136, 14-15)

b. xuškī-i dil muqāvamat natavānad kardan martarī-i jigar-rā

sec-ī-EZ coeur solidité NEG-pouvoir.PST-3SG faire-INF mar humide-ī-EZ foie-rāa le sec du coeur ne peut pas stabiliser l'humide du foie (HM 137, 1)

c. $\bar{a} n-r \bar{a} \quad b \bar{a} z d \bar{a} s ̌ t \quad$ natavānam, $\quad$ ammā tavānam $\quad$ kard $\quad k i \ldots$ cela-rā empêcher.INF NEG-pouvoir.PST-1SG mais pouvoir.PST-1SG faire.INF que... je ne peux pas l'empêcher, mais je peux faire que... (TS 114,9)

Pour $x^{v} \bar{a}$ stan, l'occurrence de HM 142, note 11 ne se rencontre que dans le manuscrit $\mathrm{F}$ et de plus le verbe régi est à l'infinitif apocopé, fait extrêmement rare dans ce texte ${ }^{32}$. Dans RA, il s'agit du seul exemple où $x^{v} \overline{a s t a n}$ exprime la volition (14). L'énonciateur associe donc une place archaïque, infinitif antéposé, à une structure archaïque, infinitif pour la volition.

\section{(14) č̀ūn dōst mar dōst-rāa sōxtan $\quad \boldsymbol{x}^{v} \bar{a} h a d$ \\ quand ami mar ami-rā brûler-INF vouloir.PST-3SG \\ quand un ami veut que son ami (litt. l'ami) brûle (RA 36, 17)}

Il est à noter que cet exemple, isolé, peut aussi s'analyser comme un infinitif nominal, objet direct de $x^{v} \overline{a s t a n}$ : «quand un ami veut la brûlure pour son ami ». Dans ce cas, on ne trouverait plus, non seulement cette place archaïque de l'infinitif antéposé après le $\mathrm{XI}^{\mathrm{e}}$ siècle, mais aussi l'infinitif avec le sens de volition dans RA. Il est néanmoins difficile de trancher ici entre infinitif verbal et infinitif nominal ${ }^{33}$.

\subsection{Structure et compléments}

Il est possible que le recours à telle ou telle structure du verbe régi soit subordonné au reste de la structure phrastique. Mais en comparant (15) et (16), on constate que les compléments n'ont aucune influence notable sur la structure choisie, quels que soient l'époque et le verbe modal : à côté de (15a) et (16a) qui emploient l'infinitif, on trouve (15b) et (16b)

\footnotetext{
${ }^{32}$ Sur les emplois de l'infinitif plein et de l'infinitif apocopé dans les textes des $\mathrm{X}^{\mathrm{e}}-\mathrm{XI}^{\mathrm{e}}$ siècles, voir Lazard 1961, p. 22 et 1963, p. 349-351.

${ }_{33}^{33}$ Je remercie très vivement Monsieur Lazard pour ses précieux conseils et pour la discussion que nous avons eue sur cet infinitif, probablement nominal.
} 
avec un verbe conjugué. En (15a) et (15b), un objet direct défini et un objet indirect dépendent du verbe régi; et en (16a) et (16b), le verbe régi est composé de kardan et d'un élément nominal avec expansion d'un objet.

(15) a. ìn pisar-rā maqām va xāna-i harām bix äham namūd ce fils-rā lieu saint et maison-EZ interdit $b i$-vouloir.PST-1SG montrer.INF je veux montrer à ce fils les lieux saints et la $K a$ 'aba (TS 48, 6)

b. $\boldsymbol{x}^{v} \bar{a}$ stam $\quad k i \quad \check{c} a p \quad \bar{u}$-rā daham vouloir.PAS-1SG que gauche lui-rā donner.PST-1SG je voulus lui donner le (sein) gauche (TS 65, 13)

(16) a. tavānad häl-i ēěs̄an-rā idrāk kardan pouvoir.PST-3SG état-EZ eux-rā perception faire-INF il peut percevoir leur état (SX 41)

b. mē tavānad ki kunad rāh namāȳ $m \bar{e}$ pouvoir.PST-3SG que faire.PST-3SG chemin action de montrer il peut montrer le chemin (SX 36)

La présence d'une relative enchâssée, précédant le verbe modal et le verbe régi, n'a pas non plus d'incidence sur la structure, avec infinitif en (17a) et verbe conjugué en (17b). Dans les deux cas, la relative, expansion au sujet ou à l'objet, précède le verbe modal. Or dans RA (17a) et TE (17b), les deux constructions sont attestées avec tavānistan et $x^{v} \overline{a s t a n}$. L'énonciateur pouvait donc opter pour l'une ou l'autre, ce qui montre bien que la relative n'a pas conditionné son choix.

(17) a. ānči man va yārān-i man bar ān-īm dalīlē vāzih bar ìn ce que moi et ami-PL-EZ moi sur cela être.PST.1PL raison-IND évident sur ce muddaā vahujjatēe lāyih barin mubtaqā mètavānad büd revendication et preuve-IND manifeste sur ce aspiration $m \bar{e}$ pouvoir.PST-3SG être.INF ce sur quoi mes amis et moi sommes d'accord peut être une raison évidente pour cette revendication et une preuve manifeste pour cette aspiration (RA 2, 16-17) 


\section{b. ìn gūnahā $\quad k i \quad$ xabar $\quad$ dădam $^{34} \quad$ bùd $\quad \boldsymbol{x}^{v} \overline{\text { ahham }} \quad k i$}

ce genre-PL que nouvelle donner.PAS-1SG être.PAS.3SG vouloir.PST-1SG que

\section{bikunam}

bi faire.PST-1SG

Je ferai ces genres (de choses) dont j'ai parlé (TE 134, 28)

Contrairement à la relative, la complétive après le verbe régi tend à faire de ce verbe un infinitif, même quand on attendrait un verbe conjugué, avec $x^{v} \bar{a}$ stan dans le sens de la volition par exemple (18). Le choix de l'infinitif évitait probablement une succession de verbes conjugués d'autant que dans les occurrences relevées, c'est finalement la complétive qui est l'élément le plus informatif de la phrase. Sans doute permettait-il aussi de ne pas accumuler les conjonctions $k i$ : dans notre corpus, seules 9 propositions avec verbe conjugué ne sont pas introduites par la conjonction, alors que la parataxe est devenue la norme en persan contemporain.
va tafsìr-aš
$x^{v} \bar{a} h \bar{\imath} m$
guftan $\quad k i \quad$ suxanhā-i $\quad i \check{s} q \quad b a$
et interprétation-ENC.3SG vouloir.PST-1PL dire-INF que parole-PL-EZ passion à
dahan-ǐ̌ān ēěān ist'imāl kunān-hand ${ }^{35}$
bouche-ENC.3PL eux utilisation faire.PPST-être.PST.3PL
et (selon) son interprétation nous voulons dire qu'ils utilisent dans leur prière (litt. dans leur bouche) des mots de passion (TE 185, 34-35) ${ }^{36}$

On peut constater en outre que l'infinitif avec $x^{v} \bar{a}$ stan auxiliaire du futur accepte autant de compléments qu'un verbe conjugué après $x^{v} \bar{a}$ stan indiquant la volition. Ainsi (19a) et (19b) possèdent le même type de compléments mais le verbe régi est à l'infinitif dans l'un et à la forme conjuguée dans l'autre. Ce choix de l'énonciateur renforce l'impression que nous avions sur la distinction de structure avec $x^{v} \overline{a s t a n}$, infinitif pour le futur, verbe conjugué pour la volition. Il apparaît clairement que les compléments comme leur nombre n’y jouent aucun rôle.

a. az nasl-i

in jamā'at

hēc ${ }^{37}$ mu'min paidā $\boldsymbol{x}^{v} \bar{a} \boldsymbol{h a d}$

$\check{s} u d$

\footnotetext{
${ }^{34}$ Cette forme paraît obscure. Nous aurions attendu dādam, «j’ai donné », sans le būd qui suit.

${ }^{35}$ On pourrait aussi lire kunānihand, passif du causatif, mais la forme est écrite en deux éléments distincts dans l'édition de Gindin.

${ }^{36}$ Cet exemple qui semble asyntaxique n'est pourtant pas une traduction mot à mot de l'hébreu : il s'agit d'un commentaire sur le texte hébreu.

${ }^{37}$ Dans la langue classique, lorsque le verbe n'est pas à la forme négative, hēč a le sens positif de «quelque, quelconque ». Nous pouvons le comparer avec le français «rien » (< latin rem, accusatif du mot «chose ») qui garde son sens positif dans certaines occurrences.
} 
de descendance-EZ ce communauté un croyant apparent vouloir.PST-3SG devenir.INF de la descendance de cette communauté un croyant apparaîtra (RA 26, 17)

b. mē $\boldsymbol{x}^{v} \bar{a}$ ham $\quad$ ki ba xudā-i tu taqarrub jōyam

$m \bar{e}$ vouloir.PST-1SG que à dieu-EZ toi accès trouver.PST-1SG

je veux être admis auprès de ton dieu (RA 37,15$)$

\subsection{Rôle de la diathèse passive}

Dans beaucoup de langues, la question de la diathèse ressortit à la morphologie. Mais ce n'est pas le cas en persan. Le passif est périphrastique : il est composé du participe passé du verbe et des formes conjuguées de l'auxiliaire šudan, «aller », «devenir », du type kušta šud, « il a été tué ». Si la diathèse a un rôle sur la forme du verbe régi par le verbe modal, c'est plus pour des raisons syntaxiques (deux formes pour le verbe régi) que pour des critères morphologiques. Dans nos textes, le verbe régi est à 5 reprises à la forme passive.

Avec $x^{v} \bar{a}$ stan, on rencontre une forme de passif dans 3 occurrences, 2 avec l'infinitif dans un sens de futur et 1 avec un verbe conjugué pour marquer la volition. La diathèse n'a donc pas d'incidence sur la structure du verbe régi par $x^{v} \bar{a}$ stan et on retrouve bien la répartition entre les deux structures : futur construit avec un infinitif, volition avec un verbe conjugué.

En revanche la diathèse passive constitue peut-être un critère de choix pour la structure régie par $b \bar{a} y a d$, «il faut». En effet les deux seules occurrences de verbe passif avec bāyad sont construites avec un verbe conjugué (20). Or excepté la diathèse, ces exemples sont en tout point semblables aux occurrences (21) construites, elles, avec un infinitif et extraites du même texte, RA.

(20) a. bāyad

ki āxir gufta šavad ba ìn tarīq

falloir.PST-3SG que dernier dire.PP devenir.PST-3SG à ce façon

il faut que ce soit dit en dernier de cette façon (RA 607, 3)

b. dar rōz-i haftum bāyad ki kušta šavad

dans jour-EZ septième falloir.PST-3SG que tuer.PP devenir.PST-3SG

le septième jour il faut qu'il soit tué (RA 619, 14)

(21) a. ba ān diraxt nazdīk namē bāyad šd

à ce arbre proche NEG-mē falloir.PST-3SG devenir.INF

il ne faut pas s'approcher de cet arbre (RA 17,2) 


\section{b. dar xasāyis-i ān hazrat suxan nabāyad kard}

dans caractéristiques.PL-EZ ce Prophète parole NEG-falloir.PST-3SG faire.INF

pour les caractéristiques du Prophète, il ne faut pas parler (RA 627, 1-2)

Les deux dernières occurrences sont à la forme négative mais nous allons voir que la négation du verbe modal n'engendre aucune conséquence sur sa construction. Seule la diathèse influencerait ici le choix de l'énonciateur. Néanmoins notre corpus présente trop peu d'occurrences pour tirer une conclusion définitive sur la diathèse passive avec bāyad.

\section{Incidence de certains morphèmes}

Comme la différence essentielle entre infinitif verbal et verbe conjugué réside dans leurs propriétés morphologiques respectives, j'analyserai ici le rôle de certains morphèmes dans le choix de la structure.

\subsection{Négation}

Cette question est à envisager sous deux angles : la négation du verbe modal et la négation du verbe régi. Palmer (2001 : 92-98) remarque que dans une majorité de langues, on peut distinguer les énoncés où le verbe modal est nié de ceux où c'est le verbe régi qui l'est. Et c'est le cas du persan qui peut nier l'un ou l'autre $(22)^{38}$.

(22) a. nabāyad

$$
\text { ki az } \bar{u} \text { fitna'e } \quad \text { mutavallid šavad }
$$

NEG-falloir.PST-3SG que de lui révolte-IND né devenir.PST-3SG

il ne faut pas qu'en naisse une révolte (PR 71, 10)

\section{b. mē bāyad ki miyān-i barādarānnizāa našavad}

$m \bar{e}$ falloir.PST-3SG que milieu-EZ frère-PL dispute NEG-devenir.PST-3SG

il faut qu'il n'y ait pas de dispute entre les frères (PR 51, 5)

Pour le verbe modal lui-même, la négation n'a aucun effet sur le choix de la structure du verbe régi. Le même verbe composé $d a f^{`} k a r d a n$ apparaît dans 2 exemples (23) à l'infinitif après tavānistan, à la forme positive ou négative.

\footnotetext{
${ }^{38}$ Pour le persan contemporain, voir les exemples donnés par Jalal Rahimian (1999: 163-167). Ces occurrences contredisent la remarque grammaticale, donc plus normative et restrictive, d'Ann K. S. Lambton (1953 : 56-57) pour qui «the negative prefix $n a$ is added to the auxiliaries $x^{v} \bar{a}$ stan, bāyestan and tavānestan (and not the main verb) if the proposition is negative ».
} 
(23) a. va tabī'at-i mafāsil

$d a f^{\prime}$

natavānad

kardan

et nature-EZ articulation.PL action de rejeter NEG-pouvoir.PST-3SG faire-INF

et la nature des articulations ne peut pas (l') éjecter (HM 34, 5)

b. $t \bar{a} \quad$ tavānad kardan

pour que action de rejeter pouvoir.PST-3SG faire-INF

pour qu'il puisse (l') éjecter (HM 106, 7)

En revanche, si l'énonciateur veut nier le verbe régi, il n'y parvient qu'à l'aide d'un verbe conjugué. En persan contemporain - mais c'est aussi vrai pour la langue classique -, Réza Mir-Samii et Pollet Samvelian (2007 : 128) ont montré que l'infinitif peut porter la négation uniquement dans son emploi nominal et jamais dans son emploi verbal; c'est une des propriétés qui les distinguent. Or, comme je l'ai rappelé, la construction infinitive des verbes modaux relève de l'emploi verbal de l'infinitif. Le corpus présente le verbe conjugué régi par le verbe modal sous la forme négative dans 5 occurrences, par exemple (22b) et (24).

$\boldsymbol{x}^{v} \bar{a}$ stam azparvadagār-i $x^{v} a d$ ki zan nakunam vouloir.PAS-1SG de créateur-EZ REFL que femme NEG-faire.PST-1SG je voulais du Créateur en personne de ne pas prendre femme (RA 631, 4)

On peut noter que ces occurrences apparaissent toutes dans PR (1 cas) et RA (4 cas), c'est-à-dire dans deux des textes les plus récents. Hasard du corpus ou évolution de la structure ? Il est difficile de trancher. Pour le persan contemporain, Azita Taleghani (2008b : 147) y voit une différence sémantique : «when the negation is sentential negation, [...] it scopes over the whole proposition. When the main verb is negated, then the NegP [...] has a narrow scope ». Dans les textes de notre corpus, seules 5 occurrences ont un verbe régi nié face à 133 exemples avec le verbe modal nié. Les premières font figure d'exception. On peut alors se demander si la nuance entre verbe modal nié et verbe régi nié existe aux premiers siècles du persan.

\subsection{Expression de la personne}

L'étude de l'expression de la personne se révèle fructueuse dans deux cas : lorsque le verbe modal ne marque pas la personne, comme pour l'impersonnel bāyad, et lorsque l'agent du verbe régi n'est pas le même que celui du verbe modal, en d'autres termes lorsqu'il n'y a 
pas coréférence entre les deux verbes. Dans les deux situations, il faut se rappeler que l'infinitif persan ne peut se fléchir en personne.

Par définition, l'impersonnel bāyad ne peut marquer une quelconque personne ${ }^{39}$. S'il entend montrer sur qui porte l'obligation, l'énonciateur peut, soit indiquer la personne avec un objet indirect marqué par la postposition $r \bar{a}{ }^{40}$ (25a), soit choisir la construction avec le verbe conjugué à la personne en question (25b).

a. $\check{s} u m \bar{a}-r \bar{a} p \bar{e} \check{s}-i \quad \bar{e} \check{a} \bar{a} n$ mē bāyad raft

vous-rā devant-EZ eux $m \bar{e}$ falloir.PST-3SG aller.INF

il vous faut aller devant eux (PR 61, 21)

b. $m \bar{e}$ bāyad $\quad k i \quad \bar{u}-r \bar{a} \quad$ girifta biyāvare

$m \bar{e}$ falloir.PST-3SG que lui-rā prisonnier $b i$-apporter.PST-2SG

il faut que tu (me) l'amènes prisonnier (PR 14,9)

L'occurrence (25b) montre la limite de la première construction : si l'énonciateur veut marquer l'objet direct défini du verbe régi avec la postposition $r \bar{a}(25 \mathrm{c})$, il lui est alors difficile d'indiquer aussi la personne sur qui porte l'obligation. C'est précisément ce que note Ludwig Paul (2002 : 26) quand il suggère que « the impersonal bāyad could distinguish the category of person more easily with $k i$ and a finite verb than with the plain infinitive ». Comme pour la négation, le choix du verbe conjugué est conditionné par la limitation des propriétés morphologiques de l'infinitif.

c. haqq-i qadīm-rāa zāya' namē bāyad guzāštan
droit-EZ ancien-rā corrompu NEG-mē falloir.PST-3SG laissser-INF
il ne faut pas laisser corrompre l'ancien droit (PR 58,9)

Une occurrence, prise hors des extraits choisis mais dans le corpus, semble mêler l'une et l'autre structure (26) pour justement éviter la confusion entre les deux compléments marqués par $r \bar{a}$. Le premier $r \bar{a}$ marque l'objet indirect, c'est-à-dire qu'il accompagne la personne sur qui porte l'obligation et le second accompagne l'objet direct défini du verbe régi. En outre,

\footnotetext{
${ }^{39}$ Je ne parlerai pas ici de $\operatorname{tavān}$, « on peut », puisque l'énonciateur choisit l'impersonnel aux formes fléchies en personnes, qui existent également.

${ }^{40}$ Gilbert Lazard (2006b : 213) rappelle que ce tour existe à époque classique mais qu'il n'est plus utilisé en persan contemporain. Helmut Satzinger (2003: 249) parle de cette tournure dans d'autres langues en la qualifiant de « elegant type ».
} 
héritage du moyen perse, le morphème - $\bar{e}$ est affixé au radical sans désinence ${ }^{41}$; c'est peut-être la raison pour laquelle l'énonciateur s'est vu contraint de mentionner la personne avec marā.

marā bāyistē $\quad k i \quad \bar{o}-r \bar{a} \quad$ zinda bidīdē

moi.rā falloir.PAS.3SG-ē que lui-rā vivant $b i$-voir.PAS-ē

il m'aurait fallu le voir vivant (litt. que je le visse vivant) (TS 333, 3)

La question de la non-coréférence entre verbe modal et verbe régi peut par ailleurs obliger l'énonciateur à recourir à un verbe régi conjugué. Ce cas ne se rencontre qu'avec $x^{v} \bar{a} \operatorname{stan}$, «vouloir », puisqu'il n'est pas possible de « pouvoir » (tavānistan) pour un autre que soi. Helmut Satzinger (2003 : 248) remarque que ce problème de coréférentialité existe dans certaines langues, comme le français «je veux que tu dises » par rapport à «je veux dire » ou l'allemand «ich will sagen » mais «ich will dass du sagst ». C'est également la situation en persan : ainsi nous avons l'exemple (27a) par rapport à (27b).

(27) a. ammā čunān $\quad \boldsymbol{x}^{v} \bar{a} h a m \quad$ ki nēkō nigāh kunēd

mais tellement vouloir.PST-1SG que bien regard faire.PST-2PL

mais je veux que vous regardiez avec attention (TS 4, 1-2)

b. ìn pisar-rā maqūm va xāna-i harām bix ${ }^{v} \bar{a} h a m \quad$ namūd

ce fils- $r \bar{a}$ lieu saint et maison-EZ interdit $b i$-vouloir.PST-1SG montrer.INF

je veux montrer à ce fils les lieux saints et la $K a$ ' $a b a(T S 48,6)$

\subsection{Le morphème bi-}

Le morphème $b i$ - peut être préfixé au verbe modal ou au verbe régi. Pour le verbe modal, seule l'analyse de $x^{v} \bar{a}$ stan est pertinente. Lorsque $b i$ - lui est préfixé, il ne provoque certes aucun changement dans la structure et n'a pas de conséquence sur le choix du verbe régi. Mais son influence est d'une autre nature: elle s'exerce sur la distinction entre volition et expression du futur lorsque le verbe régi est à l'infinitif.

Il apparaît préfixé à $x^{v} \bar{a}$ stan dans seulement deux textes de notre corpus ${ }^{42}$, tous deux datant du $\mathrm{XI}^{\mathrm{e}}$ siècle, aussi l'on ne s'autorisera à parler que de tendance de la langue. Dans TE, bi- marque à 6 reprises $x^{v} \overline{a s t a n}, 3$ avec la volition, 3 avec le futur. Cependant il faut ramener

\footnotetext{
${ }^{41}$ Lazard 1963, p. 329.

${ }^{42}$ RA présente aussi une occurrence avec infinitif et sens de futur mais cela n'est pas significatif puisque l'infinitif marque presque exclusivement le futur dans ce texte.
} 
ces chiffres au nombre plus global des occurrences contenues dans ce texte : sur 5 occurrences de volition, 3 sont marquées par bi- quand seulement 3 sur un total de 30 formes le sont pour le futur. Dans TS, la situation est similaire, 3 occurrences sont à la forme marquée, 2 pour la volition (sur seulement 4 ) et 1 pour le futur (sur 12 occurrences). Rapporté au nombre total d'occurrences, le marquage est proportionnellement plus fort avec la volition qu'avec le futur. Pour TS, j'ai déjà montré que $b i$ - fonctionnait comme indice de rhématicité ${ }^{43}$, ce que l'emploi avec $x^{v} \bar{a}$ stan confirmait : le verbe est très informatif et devient donc centre rhématique lorsqu'il exprime la volition (28a), et il a un sens plus affaibli lorsqu'il est seulement auxiliaire du futur (28b). Si ce morphème a aussi cette valeur dans TE, le marquage de $x^{v} \bar{a}$ stan obéirait à cette même logique. À l'époque de nos premiers textes, il ne s'agit encore que d'une tendance de la langue, mais celle-ci se confirmera en persan contemporain avec un autre morphème : $m i$ - se préfixe à $x^{v} \bar{a}$ stan pour la volition mais est complètement absent au futur.

(28) a. ānjā ki man bix ${ }^{v} \bar{a} h a m \quad \bar{a}$ ad

là que je $b i$-vouloir.PST-1SG aller.INF

là où je veux aller (TS 199, 3)

b. azpas-i aknūn či $\boldsymbol{x}^{v} \bar{a} h a d \quad$ rasìd?

de après-EZ maintenant que vouloir.PST-3SG arriver.INF

après cela qu'arrivera-t-il ? (TS 69, 10)

Le préfixe $b i$ - peut également marquer le verbe régi. Dans ce cas, il a une incidence sur le choix de la construction. Comme le soulignent Mir-Samii et Samvelian (2007: 118), « contrairement aux verbes finis, l'infinitif n'apparaît ni avec les préfixes aspectuo-modaux mi(préfixe de l'imperfectif) et be- (préfixe du subjonctif), ni avec les désinences personnelles verbales ». Cette caractéristique du persan contemporain se trouve déjà dans la langue classique. Lorsque l'énonciateur veut marquer le verbe régi par le morphème $b i$-, il n'a donc d'autre recours possible que d'employer un verbe conjugué ${ }^{44}$. C'est le cas dans 54 occurrences, que ce soit avec bāyad, tavānistan ou $x^{v} \bar{a}$ stan pour verbe modal. Deux variantes d'un même texte le laissent voir (29), ainsi que les deux occurrences très proches de TE (5a) et (6a). Dans ce dernier exemple, nous avions vu que les mêmes mots se retrouvaient dans les

\footnotetext{
${ }^{43}$ Lenepveu-Hotz 2008, p. 224-233, et plus particulièrement p. 232 pour $x^{v} \bar{a} s t a n$.

${ }^{44}$ Une question reste en suspens : est-ce parce que l'énonciateur veut marquer le verbe avec bi- qu'il utilise la forme conjuguée ou est-ce parce que (pour d'autres raisons ?) le verbe régi est conjugué qu'il peut ou non le marquer avec $b i-$ ?
} 
deux phrases, la seule différence se situant entre les verbes régis, vaqf būdan à l'infinitif dans la première, et la forme conjuguée préfixée de $b i-$, bišinave, dans la seconde.

a. qasd-i $\quad \bar{u} \quad \boldsymbol{x}^{v} \bar{a}$ hand $\quad$ kard mauvaise action-EZ lui vouloir.PST-3PL faire.INF

ils veulent commettre une mauvaise action contre lui (PR 43,21) majorité des manuscrits

b. qasd-i $\quad \bar{u} \quad \boldsymbol{x}^{v} \bar{a} h a n d \quad$ bikunand

$b i$-faire.PST-3PL

ils veulent commettre une mauvaise action contre lui (PR 43, 21) manuscrit $\mathrm{G}_{1}$

Le fait qu'on le rencontre également avec tavānistan indique bien que, contrairement au persan contemporain, il n'y a pas redondance de l'information : comme il n'existe pas de servitude grammaticale pour le verbe régi en persan classique, le locuteur peut choisir entre une forme non marquée (6c) et une forme marquée par bi- (6a et b).

Il en est de même si l'énonciateur veut suffixer le morphème $-\bar{e}$ au verbe régi ; 9 occurrences dont (30) l'attestent.

nabāyiste $\quad$ ki ba havā-i garmāba va havā-i čāh xaba gaštīmē

NEG-falloir.PAS.3SG-ē que à air-EZ bains chauds et air-EZ puits suffoqué devenir.PAS-1PL-ēe il ne fallait pas que nous fussions suffoqués par l'air des bains chauds ni par l'air du puits (HM $147,11-12)$

Or le marquage du verbe régi n'est pas une obligation, par exemple en (31). Si l'énonciateur choisit d'ajouter le suffixe $-\bar{e}$ également au verbe régi, c'est qu'il y a un supplément d'information, et non une servitude grammaticale.

mardumān $x^{v}$ adišān bāyistēe $\quad k i$ murtaddiān būdand

gens-PL REFL-PL falloir.PAS.3SG-ē que repentant-PL être.PAS-3PL

les gens eux-mêmes devraient être repentants (TE 179, 6)

En outre, contrairement à l'infinitif, les verbes régis conjugués peuvent varier en temps : radical du présent en (29b), radical du passé en (30). 
Ainsi constatons-nous que ce qui, dans le persan contemporain, apparaît comme redondance de marquage, surtout pour tavānistan, constitue à l'origine un véritable apport informationnel: comme il n'y a pas encore de servitude grammaticale pour le verbe régi (contrairement au persan contemporain où seul le subjonctif - avec be - est possible), un verbe régi conjugué peut porter différents préfixe et suffixe, aspectuel $(-\bar{e})$ et rhématique $(b i-)$, et peut varier en temps (radical du présent ou radical du passé).

\section{Conclusion}

Les changements essentiels dans les structures régies par les verbes modaux apparaissent au $\mathrm{XV}^{\mathrm{e}}$ siècle. L'étude quantitative des deux constructions a montré une relative stabilité entre le $\mathrm{X}^{\mathrm{e}}$ siècle et le début $\mathrm{du} \mathrm{XV}^{\mathrm{e}}$ siècle. Puis à partir de la fin de ce siècle, la structure avec verbe conjugué se rencontre plus fréquemment, à des degrés toutefois divers selon le verbe modal. L'état de langue actuel où cette construction a totalement supplanté la construction plus ancienne avec infinitif est en germe dès la fin du $\mathrm{XV}^{\mathrm{e}}$ siècle.

Pour ce qui est des causes de l'évolution, le sens de la modalité est quasi inopérant sur le choix de la structure, si ce n'est pour différencier la volition de l'auxiliaire du futur pour $x^{\nu} \bar{a}$ stan. Pour bāyistan, «falloir », et tavānistan, « pouvoir », la tendance que j'ai pu entrevoir à employer respectivement un verbe conjugué avec la modalité épistémique déductive et l'infinitif avec la modalité épistémique spéculative gagnerait à être confirmée par de plus nombreuses occurrences. D'ailleurs seules les modalités liées à l'inférence excluent l'une ou l'autre construction. En revanche avec les modalités déontique obligative et dynamique capacitive, les deux structures se rencontrent indistinctement sans que l'on puisse déceler une nuance de modalité selon la construction choisie par l'énonciateur.

Dans le domaine syntaxique, le nombre des compléments ne tient qu'un rôle très faible. On pouvait s'attendre à ce qu'un verbe conjugué permette à la structure régie par le verbe modal de recevoir plus de compléments qu'un infinitif. Mais on trouve globalement les deux structures avec des compléments similaires, quels que soient leur nombre et leur fonction. Il se pourrait que la diathèse, elle, conditionne la structure avec verbe conjugué après bāyad, « falloir », mais les occurrences manquent pour l'ériger en règle absolue.

La morphologie apporte davantage de lumière et son rôle serait déterminant : c'est à elle que l'on devrait le changement de structure. L'infinitif verbal et le verbe conjugué diffèrent essentiellement sur leurs propriétés morphologiques respectives. Comme l'infinitif verbal a des possibilités morphologiques plus limitées qu'un verbe conjugué, on comprend 
pourquoi ce dernier a pu être progressivement préféré. Un verbe conjugué peut se nier, se préfixer et se suffixer de divers affixes, tels que $b i-$ ou $-\bar{e}$ et se fléchir en personne, ce qu'un infinitif ne permet pas.

Il est enfin possible que l'évolution de la structure des verbes modaux relève pour une part du registre de langue. Cependant il est difficile de l'appréhender dans les textes persans anciens : même sans ambition littéraire, un auteur peut parfois préférer une forme écrite différente de l'expression orale qu'il utilisera dans la page suivante. On ne peut alors que supposer son influence à l'aune d'autres langues. Par exemple le français contemporain «il me faut partir » est plus soutenu que le courant « il faut que je parte », c'est le « elegant type » de Satzinger (2003 : 249). Ainsi un auteur ne pouvait-il pas privilégier une structure pour sa dimension archaïsante et donc plus littéraire, et cela en dépit du fait que les textes de notre corpus sont écrits dans une prose censée être dénuée de toute recherche stylistique?

Agnès Lenepveu-Hotz agnes.hotz@ac-creteil.fr

Abréviations : ENC : enclitique personnel ; EZ : ezâfe ; IMP : impersonnel ; IMPER : impératif ; IND : suffixe d'indéfini ; -INF : infinitif plein ; .INF : infinitif apocopé ; NEG : négation ; PAS : passé ; PL : pluriel ; PP : participe passé ; PPST : participe présent ; PREV : préverbe ; PST : présent ; REFL : pronom réfléchi ; SG : singulier.

Les particule, préfixe et suffixe $m \bar{e}, b i$ - et $-\bar{e}$ n'ont pas été glosés, tout comme la postposition $r \bar{a}$ (parfois mar... $r \bar{a}$ ).

\section{BIBLIOGRAPHIE :}

Bāteni 1354/1975 : Bāteni, Mohammed Rezā, «Este'māl-e "bāyad" dar fārsi-e emruz », in Masā'el-e zabānšenāsi-ye novīn. Dah maqāle, Tehrān : Entešārāt-e Āgāh, 1354/1975, p. 191-200. [recueil d'articles].

Brunner 1977 : Brunner, Christopher, A Syntax of Western Middle Iranian, Delmar, New York : Caravan Books, 1977.

Chantraine 1991 : Chantraine, Pierre, Morphologie historique du grec, Paris : Klincksieck, $3^{\mathrm{e}}$ édition revue et augmentée, 1991. 
Comrie 1985 : Comrie, Bernard, Tense, Cambridge : Cambridge University Press, 1985.

Creissels 1995 : Creissels, Denis, Éléments de syntaxe générale, Paris : P.U.F, 1995.

Durkin-Meisterernst 2004 : Durkin-Meisterernst, Desmond, Dictionary of Manichaean Texts.

Vol. III, Part 1. Dictionary of Manichaean Middle Persian and Parthian, Turnhout: Brepols, 2004.

Grenet 2003: Grenet, Franz, La geste d'Ardashir fils de Pâbag, Kārnāmag $\bar{l}$ Ardax̌̌èr $\bar{l}$ Pābagān, Die : éditions A Die, 2003.

Jahani 2008 : Jahani, Carina, «Expressions of future in Classical and Modern New Persian », in Aspects of Iranian Linguistics, Simin Karimi, Vida Samiian et Donald Stilo (éds), Cambridge : Cambridge Scholars Publishing, 2008, p. 153-175.

Lambton 1953 : Lambton, Ann K.S., Persian grammar, Cambridge : University Press, 1953.

Lazard 1961 : Lazard, Gilbert, «Dialectologie de la langue persane », in La formation de la langue persane, Paris : diffusion Peeters, 1995, p. 17-26. [réimpression de l'original paru dans : Našriye-ye Dāneškade-ye adabiyāt va 'olum-e ensāni-e Dānešgāh-e Tabriz, XIII (1961), p. 241-258].

Lazard 1963 : Lazard, Gilbert, La langue des plus anciens monuments de la prose persane, Paris : Klincksieck, 1963.

Lazard 2006a : Lazard, Gilbert, «More on counterfactuality, and on categories in general », Linguistic Typology, 10/1 (2006), p. 61-66.

Lazard 2006b : Lazard, Gilbert, Grammaire du persan contemporain, Tehrān : IFRI-Farhang Mo‘āser, nouvelle édition, 2006. [Bibliothèque iranienne 61].

Lenepveu-Hotz 2008 : Lenepveu-Hotz, Agnès, « Considérations sur les morphèmes hamē, -ēet bi- à l'aune d'un texte persan du XI $\mathrm{X}^{\mathrm{e}}$ siècle, le Tārīx-i Sīstān », Studia Iranica, 37/2 (2008), p. 215-240.

Mir-Samii et Samvelian 2007: Mir-Samii, Reza; Samvelian, Pollet, «Les infinitifs en persan », Faits de langues, 30 (2007), p. 117-135.

Palmer 2001 : Palmer, Frank Robert, Mood and Modality, Cambridge : Cambridge University Press, seconde édition, 2001.

Paul 2002: Paul, Ludwig, «A linguist's fresh View on "Classical Persian" », in Iran. Questions et connaissances. Volume II. Périodes médiévales et modernes : actes du IV congrès européen des études iraniennes organisé par la Societas iranologica europaea. Paris, 6-10 septembre 1999, Maria Szuppe (éd.), Paris : Association pour l'avancement des études iraniennes, 2002, p. 21-34. [Cahiers de Studia Iranica 26]. 
Phillott 1919 : Phillott, Douglas Craven, Higher Persian grammar, Calcutta: The University Press, 1919.

Rahimian 1999 : Rahimian, Jalal, «Semantic of Persian Auxiliaries », IRAL, vol. XXXVII/2 (1999), p. 153-170.

Satzinger 2003: Satzinger, Helmut, «Verbal Modality in Egyptian and Elsewhere », in Hommages à Fayza Haikal, Nicolas Grimal, Amr Kamel et Cynthia MaySheikholeslami (éds), Le Caire : Institut français d'archéologie orientale, 2003, p. 245252. [Bibliothèque d'étude 138].

Taleghani 2008a : Taleghani, Azita, «Mood and modality in Persian », in Aspects of Iranian Linguistics, Simin Karimi, Vida Samiian et Donald Stilo (éds), Cambridge : Cambridge Scholars Publishing, 2008, p. 391-417.

Taleghani 2008b : Taleghani, Azita, Modality, Aspect and Negation in Persian, AmsterdamPhiladelphia: John Benjamins Publishing Company, 2008. [Linguistik Aktuell/Linguistics Today 128]. 